\title{
The Development and Application of Extensible Computer Network Design System
}

\author{
Wang Yi, Li Liang \\ Shandong Labor Vocational and Technical College, Jinan Shandong 250022, China \\ Shandong Computer Science Center, Jinan Shandong 250014, China
}

Keywords: Extensibility; Computer network design; Software system; Development design

\begin{abstract}
The mature development of computer network technology mainly is due to the combination with OSI network model's feature of continuous software improvement. In the analysis process of software life cycle, improve the practicability of the software. This paper focuses on analyzing the development and application of extensible computer network design system.

Researches about computer network design software system are various at home and abroad. Foreign scholars put forward that computer network software system design process needs comprehensive consideration of topology structure, analysis of router security as possible and comprehensive analysis of the capacity's safety expression. Make an extensive application of computer network design to extend the service life of the software and, in turn, increase the functions of the software and promote large-scale software construction and development [1]. The application of computer network design pays attention to visual function optimization extension, and in the development of science and technology, constantly updates network technology structure. Software analysis function extension combines with the upgrading form of network technology, makes full use of the latest network performance model and at the same time, makes the scientific optimization design of the project so as to actively build new network performance model [2]. This article mainly makes use of software external interface extensibility research to promote networked office and application. At the same time of network software pin application, do a good job in data protection and promote the basic extension of computer network software. The design and development process of extensible computer network design system mainly considers topology structure to put forward an extensible network design software system, and promote large-scale development of network technology. Gradually integrate the new plug-in form in software system structure.
\end{abstract}

\section{THE DIRECTION OF EXTENSIVE COMPUTER NETWORK DESIGN SOFTWARE SYSTEM}

In general, software extensibility, as software worker's goal, can effectively extend software life cycle. This extensibility can not only be embodied in software size extensibility, but also be embodied in software function extensibility. Based on extensive computer network application, it shows a kind of unique function extensibility. This application stage of computer network technology has three different directions.

A. Visual software function extensibility

Visual software function extensibility embodies itself in new network product features through innovation network technology function pattern, and it can effectively track the development of network technology at the same time, combined with the embodiment of software network protocol function.

B. Performance analysis ability extensibility

Based on performance analysis stage, combined with large-scale development of network technology, do a good job of network optimal design and application. A new performance model structure makes comprehensive evaluation for the project design. Based on convenient application of software application ability, build a new performance model structure [3].

C. Software external interface extensibility 
While extend external interface software, mainly complete network design project analysis. Strengthen the standardization management of network equipment and at the same time maintain network design project. Complete software optimization design, optimize interface, and realize the extension of the interface.

\section{THE STRUCTURE DESIGN OF EXTENSIVE COMPUTER NETWORK DESIGN SOFTWARE SYSTEM}

Computer network design process needs the design of software function modules, as shown in Figure 1.

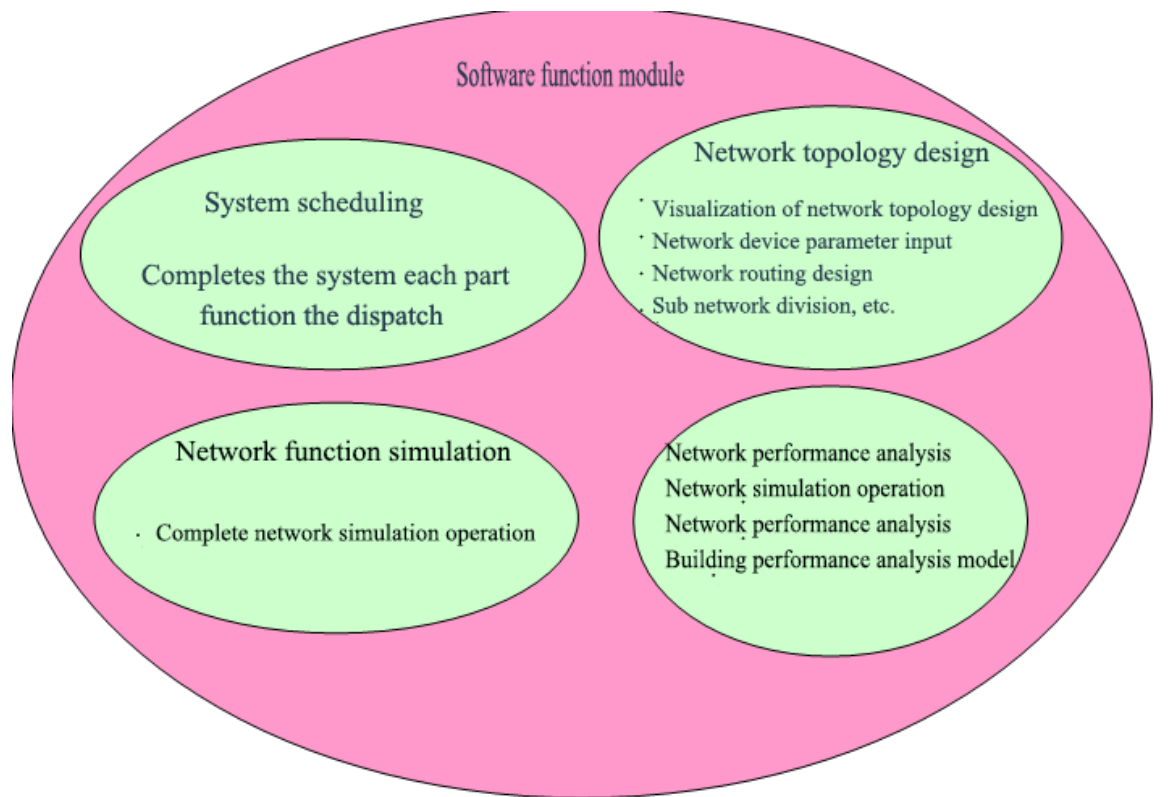

Figure 1 Software function module

Software function module is mainly to do a good job of system scheduling to complete some functions' dispatching work. Network topology design process realizes a visualized network topology design to do a good job in effective input and analysis of network equipment parameters at the same time of network equipment parameter input. The basic design and application of network routine do a good job of sub-net division. Network performance simulation process realizes the simulation calculations and analysis [4]. For network performance analysis, combine with network simulation calculations and analysis, make network performance analysis process, and establish performance analysis model.

While divide different layers of software, combine with network device object and the effective application of equipment parameters, and make good analysis of operating system structure modules in coordination software application stage. Visualized network topology design process realizes network simulation and performance analysis application. It embodies software application function and makes good application of software function structure. The connection and application of database and software system, combined with a variety of application modes with hierarchical structure, pay attention to the relevant interface analysis of network equipment. The functional modules characteristics pay attention to the application of multi-layer software architecture and make large-scale connections and analysis of software system. Same structure's application process under the software does a good job in the application of network equipment and promotes the equipment parameters' interface optimization [5]. Some of software's independence function with the function feature of network equipment completes the effective call and processing of software functional. This function's call process realizes the sharing of data and makes the sharing of data application. Data access timely resolve phase, combined with functional application of sharing data, improves equipment class library. Software system structure diagram is shown in Figure 2. 


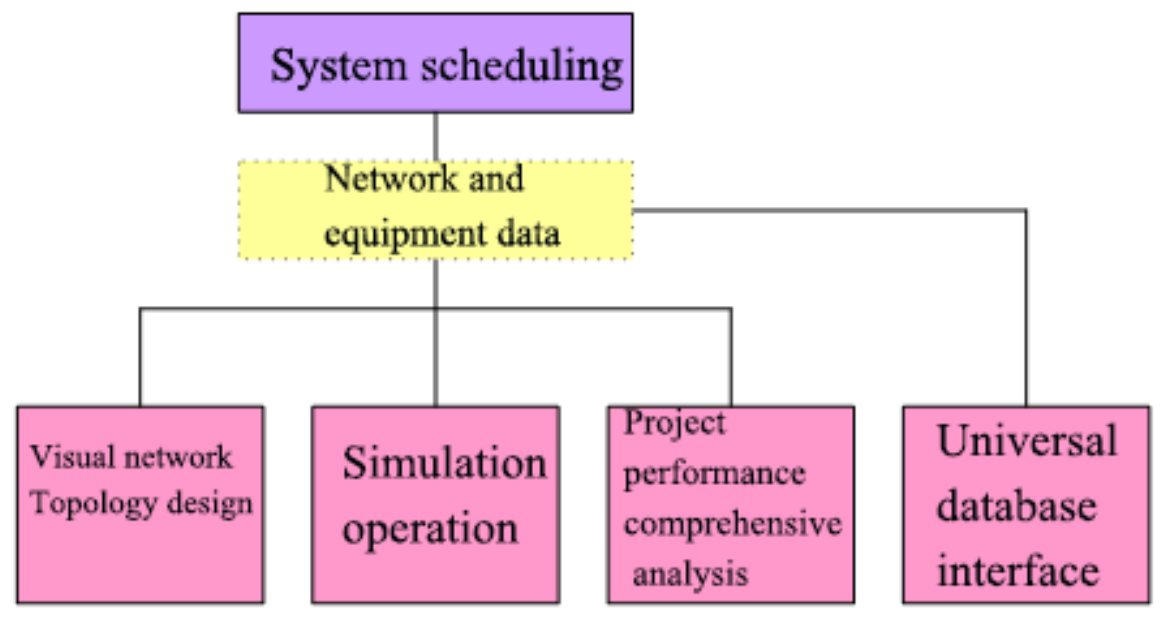

Figure 2 Software system structure

\section{THE SOFTWARE SYSTEM DATA INTERFACE AND OBJECT OF EXTENSIVE COMPUTER NETWORK DESIGN}

System data software is put forward with the combination of the unique function extension process, and ensures a fixed object interface under the application of software object internal structure. It determines the unique software application architecture and promotes the optimization and application of software data interface.

A. Problem description

Network software design is mainly to do a good job of editing and processing network topology. In network design phase, set up and remove devices to achieve equipment division, and then make good sub-net construction and management [6]. Network configuration process makes use of the optimized configuration and application of computer network for project rational design and achieves the optimal design and application of the database. Equipment display process is to make equipment configuration and effective simulation process.

B. Identification of classes and objects

Identification of classes and objects mainly completes the effective analysis and application of the object. In the realization of software structure, it reflects unique extension ability. System scheduling process completes network topology optimization design. The application of network simulation pays attention to optimization analysis of performance, and then makes simulation calculation, performance analysis, and function embodiment. With software function extension, make large-scale application of network device object, and establish reasonable network classes and equipment [7].

Network topology design process response is shown in Figure 3. 

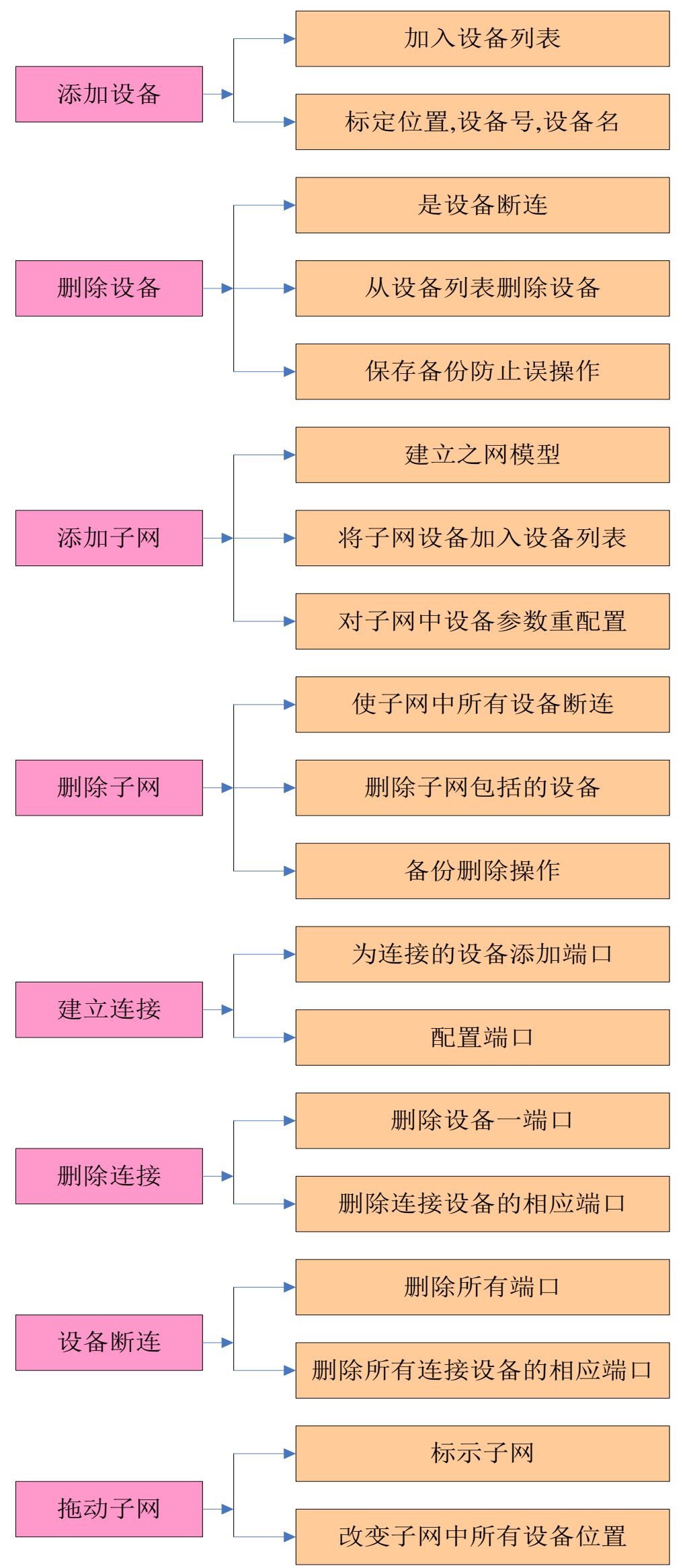

Figure 3 Network topology design process response

Simulation phase is mainly to initialize the network, set up event table, complete the simulation operation work, improve the dynamic display network of operation process, and achieve the optimization analysis and application of result performance. While identify network equipment's 
initial state, establish operation period, do scanning of other device status and calculate the time when equipment state changes.

C. Elaboration classes and objects

1. Establish standard framework model

In general, network device has many equipment types. Based on the expansion process of the software, do a good job in the application and analysis of standard model, combining with international standardization organization mode; make a standard framework structural construction of the network. Based on the application of equipment model, with a perfect network structure, make device data structure's basic application and pay attention to the embodiment of network equipment function. The application of network level pays attention to the validity definitions of software device and shows unique model function characteristics application. While refining class and object, combine with OSI model and pay attention to standard framework model establishment. In the application process of consummation network equipment data structure, pay attention to the effectiveness application of network equipment and manifest the unique physical structure. Software device class model definition is necessary to reflect model feature uniqueness, makes the performance application of simulation budget, and then completes the grammar description, which is shown below:

class CDevice

\{

protected :

$\mathrm{m}$ - deviceport;

$\mathrm{m}$ - deviceposition;

$\mathrm{m}$ - devicename;

int $\mathrm{m}$ - deviceprice;

int $\mathrm{m}$ - devicetype;

int $\mathrm{m}$ - runstate;

intm — showstate;

int $\mathrm{m}$ - deviceid;

\}

2. Setting up equipment

Setting up equipment classes is mainly to set scale model application. Pay attention to the effectiveness collection of the device object and reflect the large-scale application of network data model. It is shown below:

class CNet

\{

private:// $\square \square \square \square \square \square \square \square$

$\mathrm{m}$ - netsize; // $\square$

$\mathrm{m}$ - focusdevice;//

$\mathrm{m}$ — netdevice;//

\}

\section{Equipment port connection}

The expression of equipment port is mainly in accordance with the basic connection process of device physics. With performance parameters application, reflect best position characteristics, make rational analysis of equipment port model, and realize the effectiveness connection with the device. An application based on simulation process pays attention to the direct analysis of performance, and realizes the related data expression of the port class model, combined with port class model application, which is described as: 


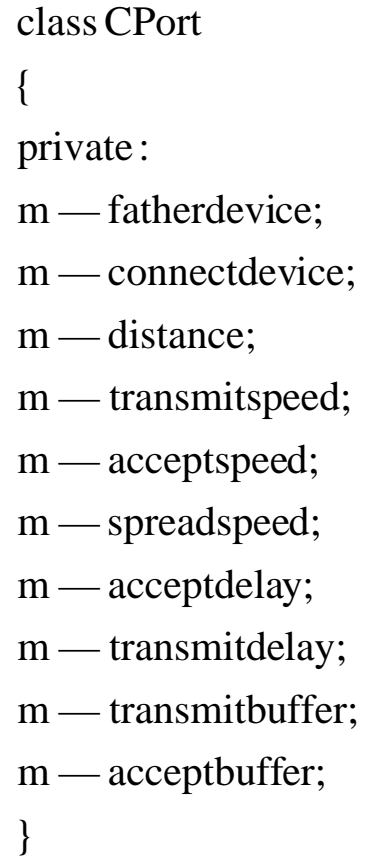

4. Simulation

The process of simulation calculation is mainly in combination with the connection between different equipment and pays attention to effective data flow. The relevant data application of message model is shown below:

struct sMessage

\{

$\mathrm{s}$ - sourceaddress; // $\square$

$\mathrm{s}$ - destinationaddress;// $\square$

$\mathrm{s}$ - type;//

s - length;//

$\mathrm{s}$ - transmittime; //

$\mathrm{s}$ - abandontime; //

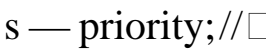

\}

The embodiment of data model characteristics, combined with various development and application of network technology, realizes network equipment simulation process and pays attention to the large-scale analysis of the performance. Based on the analysis of database related interface, combined with a variety function models of data structure, make application of software basic data interface.

\section{THE ESTABLISHING CONDITION AND FUNCTION EXTENSION OF EXTENSIVE COMPUTER NETWORK DESIGN SOFTWARE SYSTEM}

\section{A. System establishment conditions}

The establishment of the system needs to combine the basic structure characteristics of the system, and mainly analyzes the functional interface situation of the data. In system topology analysis stage, do a good job of effective add and deletion of the system. Mt net bus application, combined with simulation calculation's functional applications, realizes the function features of the software. The Mt net bus function model application process fundamentally realizes the effective control of software, and embodies function extension and analysis of software uniqueness. The switches' function characteristics in software exchange and application realize the design of system 
scheduling module and realize the functional extension.

B. System function extension

The realization of software extension function not only does a good job in the perfection of network equipment library, but realizes the effective extension of database interface in accordance with more reasonable and effective simulation calculation process and paying attention to performance effectiveness analysis application. Based on the application of formal network description language, it has a convenient function extension, which implements a simple code processing with software function optimization design. Results show that extensible computer network design software system development and design can satisfy the increasing demands of people's intelligent communication, which increases the efficiency of computer network applications. The system has stable operation, and this promotes the development of computer network technology to be more perfect and mature, and provides convenient conditions for the rational use of computer network in our country.

\section{CONCLUSION}

The function characteristics improving process of computer network design software reflects the design of reasonable equipment model. Design many forms of network description language and promote the deep design and development of computer network in our country to a higher study level. The article is intended to comply with people's increasingly higher requirements for intelligent communication, improves the effectiveness of computer network application, and reasonably designs computer network application system. By offering an extensive computer network design software system, analyze computer network design software system knowledge structure, and put forward a kind of object-oriented method network design process. Extensible computer network design software system development and design can satisfy people's increasingly high intelligent communication demands and increase the efficiency of computer network applications. The system has stable operation, and this promotes the development of computer network technology to be more perfect and mature, and provides convenient conditions for the rational use of computer network in our country.

\section{REFERENCE:}

[1] Guo Li. Packet Tracer software's practical application research in computer network experiment teaching [J]. Computer CD Software and Applications, 2014, 11 (1) : 222-223.

[2] Sun Kairong. Object-oriented extensible computer network software system design [J]. Computer Knowledge and Technology, 2014, 12 (3) : 540-541.

[3] Li Yang. Object-oriented extensible computer network software system design [J]. Computer CD Software and Application, 2013 preceding (24) : 61-64.

[4] Liu Ligang, Huang Jing. Web IOU software application in computer network experiment teaching [J]. Modern Computers (Professional Edition), 2013, 06 (9) : 56-58.

[5] Yu Runzhong. Introduction to simulation design software application in computer network foundation course [J]. Chizi, 2012, 06 (9) : 105-144.

[6] Yu Jianrong. Application research on computer network software [J]. Computer CD Software and Applications, 2014, 23 (24) : 79-80.

[7] Zhang Weihong. Object-oriented extensible computer network design software system research [J]. Journal of Digital Communication World, 2015,07 (9) : 58. 